Journal of

Molecular Microbiology

and Biotechnology
J Mol Microbiol Biotechnol 2012;22:335-343

DOI: $\underline{10.1159 / 000345328}$
Published online: December 5, 2012

\title{
The Mimic Chain Reaction
}

\author{
Vic Norris $^{a}$ Alain Thierry ${ }^{b}$ Patrick Amarc I. Barry Holland ${ }^{d}$ Franck Molina $^{b}$ \\ ${ }^{a}$ Theoretical Biology Unit, EA 3829, Faculty of Science, University of Rouen, Mont-Saint-Aignan, ${ }^{b}$ Sysdiag UMR \\ 3145-CNRS/BIO-RAD, Montpellier, ' LRI UMR CNRS 8623, Université Paris-Sud and AMIB Project, INRIA Saclay, and \\ d Institut de Génétique et Microbiologie, UMR CNRS 8621, Université Paris-Sud, Orsay, France
}

\section{Key Words}

PCR • Autodisplay • Autocatalysis • Quorum sensing • Immune system - Ligand - Receptor - Secretion - Epitope • Two-component system

\begin{abstract}
It is sometimes speculated that the equivalent of the polymerase chain reaction might be developed for identification of peptides, proteins or other molecules. In general, though, it is believed that there can be no way to amplify targets such as proteins. Natural amplification systems do, however, exist as in the case of certain autoinducer systems in bacteria. Here, we outline a possible, generic method, the mimic chain reaction, for obtaining peptides with 3-D structures that mimic the 3-D structure of their targets. These targets would include a variety of molecules, including proteins. There are therefore two categories of applications: the ability via amplification firstly to detect a known protein or other target at an extremely low concentration, and secondly to obtain a set of peptides that mimic the structure of an unknown target and that can be used to obtain a 'photofit'.
\end{abstract}

(C) 2012 S. Karger AG, Basel

$1464-1801 / 12 / 0225-0335 \$ 38.00 / 0$

Fax +41613061234

E-Mail karger@karger.ch

www.karger.com
Accessible online at: www.karger.com $/ \mathrm{mmb}$

\section{Introduction}

An amplification system for a wide variety of molecules and macromolecules might be envisaged that would be analogous to the polymerase chain reaction (PCR) for the amplification of nucleic acid sequences. This would allow the detection of molecules in vanishingly small amounts and might even allow the quantification and structural analyses of these molecules. It might draw its inspiration from existing, well-understood systems such as quorum sensing, two-component systems and autodisplay. Quorum sensing or efficiency-sensing or the less teleological auto-induction is used by bacteria to coordinate their behavior across a population in a density-dependent way [Hastings and Greenberg, 1999; Hense et al., 2007]. Many different molecules can be used as signals. These include $\mathrm{N}$-acyl homoserine lactones in Gram-negative bacteria, oligopeptides in Gram-positive bacteria and autoinducers in both Gram-negative and Gram-positive bacteria. As the population density increases, the concentration of the inducer passes a threshold, which leads to still more inducer being synthesized. This positive feedback results in the full activation of the receptor and the upregulation of specific genes under its control throughout the population. The classical illustration of this is production of bioluminescence by Vibrio fischeri,

\footnotetext{
Vic Norris

Theoretical Biology Unit, EA 3829, Faculty of Science

University of Rouen

FR-76821 Mont-Saint-Aignan (France)

E-Mail victor.norris@univ-rouen.fr
} 
which would not be visible if the luciferase responsible were synthesized only by a single cell. In the model Gramnegative Escherichia coli, auto-inducer-2 is produced and processed by the $l s r$ operon which also encodes an $\mathrm{ABC}$ transporter that imports AI-2 during the early stationary phase of growth. AI-2 is then phosphorylated by the LsrK kinase to yield phospho-AI-2, which in turn activates the lsr operon by suppressing a repressor, LsrR [Turovskiy et al., 2007]. Systems affected by AI-2 include the two-component $q s e B-q s e C$ operon which plays an important role in biofilm formation [Yamaguchi et al., 2009].

Two-component systems are based on a sensory histidine kinase (a transmitter) and cognate response regulator (a receiver) This is the case of the staphylococcal agr locus, which also encodes a quorum sensing system controlling the expression of virulence via a two-component system. Operation of the agr system entails secretion of a peptide, AgrD, in a modified form as an autoinducing peptide of seven to nine amino acids with a thiolactone ring [Novick and Geisinger, 2008]; this autoinducing peptide can bind to a membrane-bound receptor, AgrC, which then phosphorylates the response regulator, AgrA, to activate transcription of the agr operon containing the $\operatorname{agr} A, \operatorname{agr} B, \operatorname{agr} C$ and $\operatorname{agrD}$ genes. The ability of an autoinducing peptide to activate its cognate receptor is highly sequence-specific and substitution of a single amino acid can change specificity. The agr system is particularly interesting for developing a general amplification system as it has been suggested that "agr autoactivation, unlike classical enzyme induction, can occur under suboptimal conditions and can distinguish self from non-self by inducing an exclusive and coordinated population wide response" and, moreover, that a "sharp activation threshold is seen with autoactivators" [Novick and Geisinger, 2008].

Two-component systems are considered a precious resource for synthetic biology [Ninfa, 2010]. This is because these systems are functionally isolated from one another due to the high specificity of (1) the interactions between sensor/transmitter and cognate response regulator [Skerker et al., 2008], and (2) the phosphatase activities of these systems [Carmany et al., 2003; Perego, 1998]. Moreover, such systems can be introduced into other species that have no analogous system. Moreover, hybrids can readily be made between different systems and, for example, it has proved possible to fuse the Tar chemoreceptor protein and the EnvZ sensor kinase to give a hybrid protein that can sense the level of aspartate (which is normally sensed by Tar) and transduce this information so as to regulate a gene under the control of EnvZ [Utsumi et al., 1989].
Autodisplay has been defined as the surface display of recombinant proteins or peptides by means of an autotransporter, a system that Gram-negative bacteria use to transport proteins to the cell surface or secrete proteins into the extracellular milieu. Autodisplay can allow over $10^{5}$ peptides per cell to be displayed on the surface of the bacterium without seriously reducing viability. This is important if FACS is to be used to select specific subpopulations of bacteria. For example, autodisplay of a random library of peptides, some of which bind to an enzyme of interest, tagged for detection by FACS, has been used to obtain three new peptide inhibitors of cathepsin G [Jose et al., 2005].

Inspired by two-component systems, oligopeptide autoinducers, autodisplay and the PCR for the amplification of nucleic acid sequences, we discuss here the likely future development of an amplification system for molecules other than DNA and RNA. We describe a generic amplification system for detecting at extremely low levels both known molecules/macromolecules (such as prions) and unknown molecules (such as the tumor-derived nucleotides and other molecules circulating in the bloodstream [Mouliere et al., 2011]); the components developed in this system may themselves be useful for the quantification and structural analysis of the molecules of interest. We outline strategies to construct such systems with the intention of inspiring discussions among readers about how to improve these strategies and, ultimately, about how to achieve generic amplification.

\section{Principle of the Mimic Chain Reaction}

There are four components in the MCR: the target, which can be either known or unknown; the receptor, which can bind to part of the target and which, when binding occurs, generates a signal; the mimic, which has a structure sufficiently similar to part of the target as to act as a ligand to the receptor in place of the target; the amplifier, which contains both the regulator that responds to the signal from the receptor and the regulated machinery that makes the mimic. The target can be a protein or other molecule, including small molecules. The mimic can be any protein or peptide that can be encoded and that adopts a wide variety of structures (note that DNA and RNA aptamers could also be used). The amplifier can be a cell, liposome or other container; if the amplifier is a cell, the receptor can be a membrane protein, the regulator can be another protein and the regulated production machinery could be a gene encoding the mimic. 


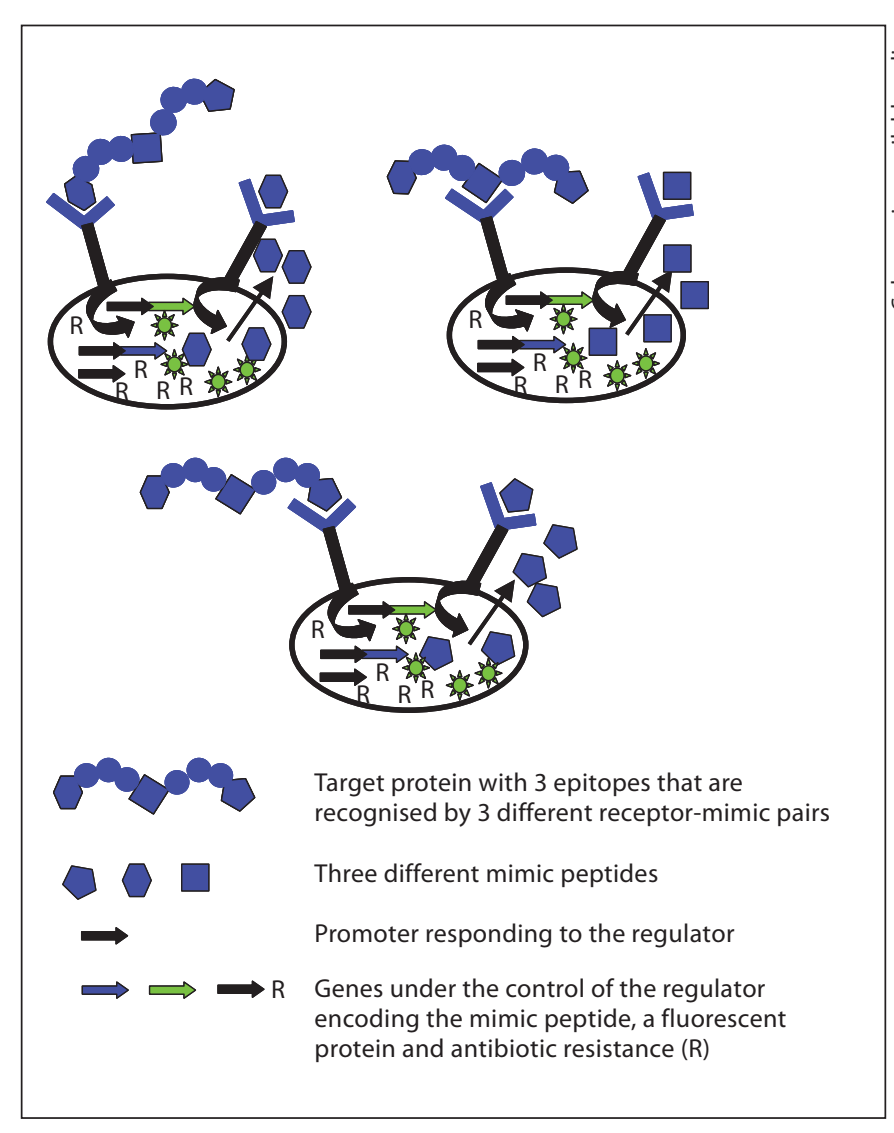

Fig. 1. Binding of the target protein to a receptor stimulates production of the mimic peptide, antibiotic resistance and fluorescence.

MCR operates in the following way. The target binds to a receptor that then signals to the regulator to promote synthesis of the mimic. The mimic is then exported and binds to more of the same type of receptor. This is a positive feedback process that could allow, in principle, detection of a single copy of virtually any molecule (fig. 1). Consider one, specific implementation of MCR. A library is constructed of bacteria each with a membrane-bound receptor with a different extracellular domain (for example, similar to AgrC) and containing a regulator (similar to AgrA) and regulated production machinery in the form of a gene (like $a g r D$ ) encoding a mimic peptide plus appropriate transcription, translation and export machinery. All these RM (for receptor mimic) bacteria have a receptor that binds to the mimic peptide that it produces, i.e. all RM bacteria have a cognate receptor-mimic pair.

A target protein can be considered as a set of antigens each of which can bind to a different receptor. When MCR is used to amplify a known target protein, the RM bacteria used all have a receptor that can bind to the tar-

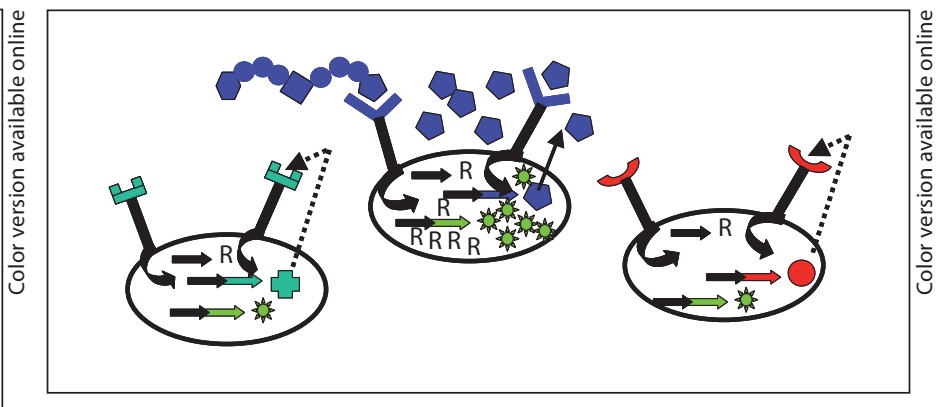

Fig. 2. Detection of an unknown protein at a low concentration by a library of RM bacteria containing a large number of different cognate RM pairs. The solid arrows indicate auto-induction triggered by the protein whilst the dotted arrows indicate the unrealised possibility of auto-induction. Other symbols as in figure 1.

get (and to the corresponding mimic that they produce) although different bacteria in this population may have different receptor-mimic pairs. When MCR is used to amplify an unknown target protein, a library is used in which only a few of the RM bacteria have receptors that can actually bind to this target because the vast majority of these bacteria are able to bind to other possible targets (fig. 2).

In the case of an unknown target protein, suppose a few of the RM bacteria in the library bind to it. If the same circuit that switches on the autocatalytic production of the mimic peptide also switches on resistance to an antibiotic and/or fluorescence, these bacteria can be selected because only they can grow in the presence of the antibiotic or because they can be separated from the rest of the population on the basis of their fluorescence. These bacteria encode a set of mimic peptides that are a partial copy of the target protein itself, the sequences of these peptides reflect those in the target protein, and the sequences of the receptors correspond to those of antibodies to the target protein. This information can be used to characterise and identify the protein (see 'Structural insight'). Moreover, this subset of the original RM bacteria constitutes a population that can now be used to detect the same or related proteins in the future.

\section{Library Construction}

The MCR method entails constructing a bacterial library of cognate pairs of surface receptor/exported mimic peptide (fig. 3). 

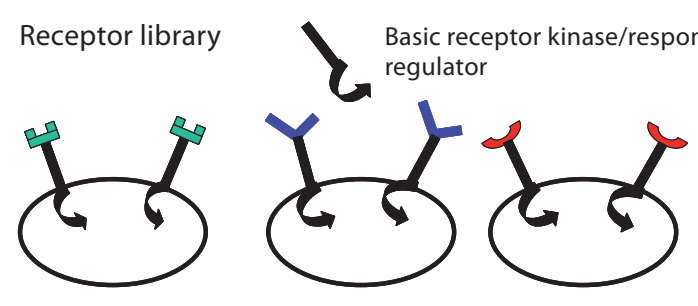

Peptide library
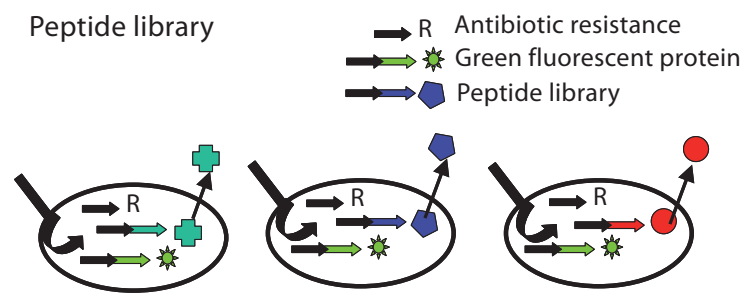

a

The population of bacteria containing the receptor library, $\mathrm{Rx}$ bacteria, are transformed with the basic plasmid containing ONLY the inducible gfp.
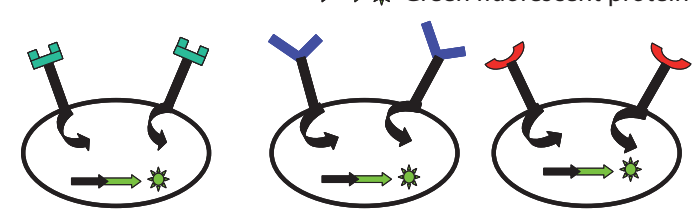

An excess of the target protein is added to the highly varied $R x$ bacteria and those bacteria that fluoresce are selected (e.g. via flow cytometry). This subpopulation of fluorescing Rx bacteria is then re-examined in the absence of the target protein and those that still fluoresce are discarded. Repeat etc.
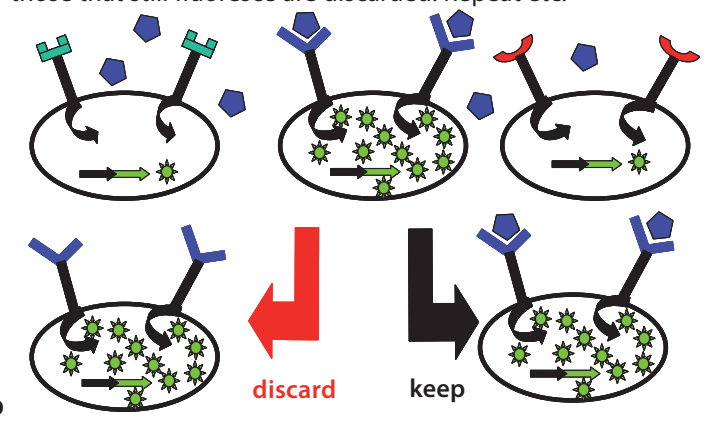

The Rx subpopulation that only fluoresces in presence of the target protein is then retained. This is then transformed by the plasmids containing the mimic peptide library.

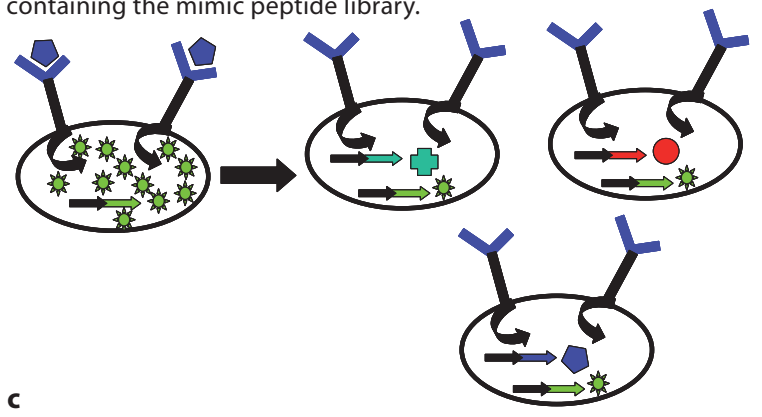

A very low concentration of target protein is then added, of the order of one molecule per cell, to select for those bacteria with a receptor-mimic pair that activates the autocatalytic circuit. The bacteria are then plated on the antibiotic to which only bacteria that have an active circuit are resistant.
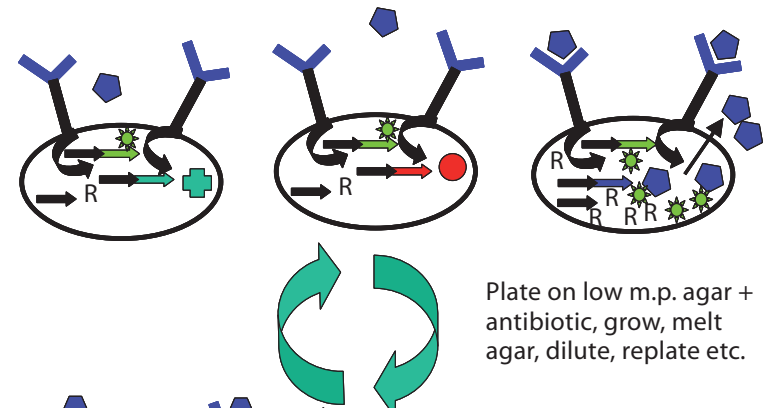

Plate on low m.p. agar + antibiotic, grow, melt agar, dilute, replate etc.
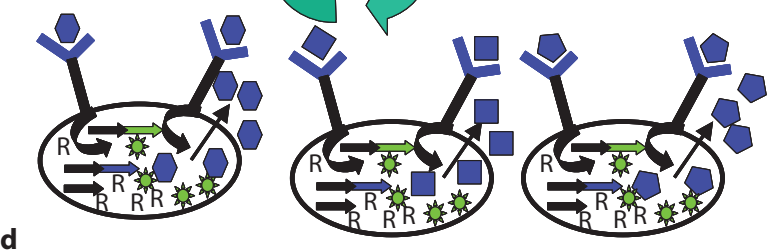

Fig. 3. Construction of the RM library. a Construction of separate receptor and peptide libraries. $\mathbf{b}$ Selection of a subpopulation of the receptor library that only fluoresces in the present of many copies of the target protein. c Transformation of this receptor subpopulation by the mimic peptide library. $\mathbf{d}$ Selection of those bacteria that fluoresce in the presence of a few copies of the target protein. 


\section{Step 1}

Construction in vitro of the MCR receptor library. As with construction of a phage library, a sequence of DNA is mutagenized and these sequences are then ligated in place of the usual extracellular sensing domain of a membrane-bound sensor kinase (e.g. of the EnvZ type). In the case of receptors and mimics (see step 3), it may be important to subject the mutagenized DNA to a 'filter' to remove sequences encoding relatively unstructured peptides or those that can adopt many conformations. Such filters might be based on ultra-centrifugation, on electrophoretic separation or on binding to macromolecules extracted from a wide range of cells from across the phyla so giving maximum coverage of shape space [Perelson and Oster, 1979]. It may prove useful to run preparative 2 -D gels, stain them and measure the amount of protein, then to cut them into, e.g. $50 \times 50$, small squares and to use much less protein from the more densely stained spots so as to prevent over-representation of abundant proteins.

\section{Step 2}

Introduction of the MCR receptor library into a bacterial population. This is achieved via one of the standard methods (e.g. electroporation and selection for resistance to an antibiotic).

\section{Step 3}

Construction in vitro of a mimic peptide library. $\mathrm{Mu}-$ tagenized DNA sequences encoding mimic peptides (e.g. similar to the 12 residue peptide that binds at low nanomolar affinities to the oncogenic proteins MDM2 and MDMX [Pazgier et al., 2009]) are ligated behind the promoter region of a gene regulated by the response regulator (e.g. of the OmpR type) and are inserted into a selectable plasmid. This plasmid also contains an identical promoter region in front of the $g f p$ gene encoding the green fluorescence protein, GFP, and in front of the gene encoding an antibiotic resistance. A basic plasmid is also constructed that only contains this promoter region in front of $g f p$ and the resistance gene.

\section{Step 4}

The population of bacteria containing the receptor library, Rx bacteria, are transformed with the basic plasmid containing ONLY the inducible $g f p$.

\section{Step 5}

An excess of the target protein is added to the highly varied $\mathrm{Rx}$ bacteria and those bacteria that fluoresce are selected (e.g. via flow cytometry). This subpopulation of fluorescing Rx bacteria is then re-examined in the absence of the target protein and those that still fluoresce are discarded. This process is repeated as many times as necessary to obtain bacteria that only fluoresce in the presence of the target protein.

\section{Step 6}

The Rx subpopulation that only fluoresces in presence of the target protein is then transformed by the plasmids containing the mimic peptide library. (It may be necessary to have a selection to eliminate the basic plasmid, perhaps on the basis of incompatibility.)

\section{Step 7}

A very low concentration of target protein is then added, of the order of one molecule per cell, to select for those bacteria with a receptor/mimic pair that activates the autocatalytic circuit. The bacteria are then plated on the antibiotic to which only bacteria that have an active circuit are resistant. (Alternatively, this could be done using the fluorescence approach as in step 5 above.) This should give a set of bacteria with different receptor/mimic peptide pairs to the target protein.

\section{Implementation}

The ability of an autoinducing peptide to activate its cognate receptor is highly sequence-specific and substitution of a single amino acid can change specificity. In principle the agr system itself might be used, although in reality this system is probably too complicated. First, an agr operon should be constructed that can operate in a model bacterium such as B. subtilis or even the Gramnegative $E$. coli. Second, the DNA encoding the domain from AgrC that binds the autoinducing peptide should be heavily mutagenized. This now constitutes the Receptor Library (fig. 1). The mimic peptide library could be constructed starting with a standard phage library and selecting those phages that bind to the entire AgrC-based, receptor library and discarding those that bind to the unmodified AgrC. The DNA extracted from this phage would be ligated into the agr operon in the place of agrD. This is not enough since AgrB, which is in the membrane, and AgrD normally combine to generate the activating ligand, a 7-9 amino acid peptide that contains a 5 amino acid thiolactone (or, in one case, a lactone) ring. So an altered AgrB would have to be selected that would export the AgrD-based, peptide library. 


\section{Additional Advantages}

\section{Structural Insight}

The structural analysis of a set of cognate receptormimic pairs by, for example, NMR may prove precious insights into the structure of the region of the target binding to the receptor. This is because the structures of the individual mimic and complementary receptor can be obtained separately and then this information can be combined and refined. Moreover, there may be more than one receptor-mimic pair for the same epitope. Putting together the structures of receptor-mimic pairs to different epitopes may allow a photofit to be obtained that would help characterise the target. Note here that these structural insights give MCR an additional advantage over mass spectrometry.

\section{Detection of Modified Forms of the Same Molecule}

Macromolecules may undergo scores of post-translational modifications including well-known ones such as glycosylation, phosphorylation and methylation as well as lesser-known ones such as addition of poly(R)-3-hydroxybutyrate. These modifications often result in significant changes in conformation that affect, for example, differentiation and signaling. In principle, MCR could generate a spectrum of RM pairs to a whole range of conformations including those that depend on the modification. Hence, the difference between spectra to a modified and an unmodified protein might not only show that the protein undergoes a particular modification but also give information about the structural consequences of that modification.

\section{Quantification}

Antibodies are routinely used in Western blots to quantify the amounts of their targets in different samples. If an excess of purified receptors (obtained via MCR) to the target were used, it should prove similarly possible to determine the amounts of a target in different conditions.

\section{Simultaneous Detection of Different Targets}

Second-generation developments of the system should include extending it to cover a wide variety of types and, in particular, sizes of molecules, and to detect simultaneously several different targets in the same sample. The latter might be achieved via the construction of different RM libraries each using a fluorescent protein such as GFP, EBFP or YFO.
Kits

MCR lends itself readily to the development of kits and one can anticipate that the MCR will be relatively cheap and easy to do in many laboratories.

\section{What Are the Problems?}

\section{Noise}

A highly sensitive library of RM bacteria capable of detecting only one protein should be expected to be noisy and give false-positives. Reliability might be improved by increasing the levels of the phosphatase that dampens the phosphate transfer at the heart of sensor kinase/response regulator signalling. Reliability might also be improved by requiring the receptor to work only as a dimer with each heterologous monomer binding to a different epitope on the same signal target protein; alternatively, the same cell might contain two independent receptors, the activity of both being needed to switch on production of two mimic peptides in that cell. The feasibility of constructing heterologous dimers is indicated by autodisplay experiments using the AIDA-I adhesin from Escherichia coli which revealed a dimerisation driven by the displayed protein, SDH [Jose and Meyer, 2007]. Presence of a low concentration of a known target protein might best be indicated by the fluorescence of many different receptormimic pairs which would require the use of different versions of GFP (see above).

\section{Mutants}

Bacteria have a formidable propensity to mutate, recombine and rearrange their DNA. The resulting genetic changes might lead to spontaneous fluorescence and resistance in the absence of binding by the target protein to a specific receptor. To reduce recombination and rearrangement, it may be worth using bacteria such as the reduced-genome $E$. coli that lacks certain recombiogenic elements [Posfai et al., 2006].

\section{Nonspecific Binding}

The binding of the target protein to molecules for which there is no specific recognition is likely to lengthen the time taken to encounter one of the rare, highly specific receptors in the RM library. How long may the protein spend bound to non-cognate receptors and other molecules and how will this limit the sensitivity of MCR? The lac repressor spends around $90 \%$ of time nonspecifically bound to and diffusing along DNA with a residence time of $<5 \mathrm{~ms}$ [Hammar et al., 2012]. In the construction 
of the library (step 7, above), the exact ratio of target:cell may have to be determined experimentally for each target. Using a 1:1 ratio risks some cells not being visited whilst using a high ratio risks artifactual stimulation of a bacterium because of the simultaneous, nonspecific binding of several copies of the target to receptors that would not normally be recognised.

\section{Diffusion}

The time taken for the target protein to visit every bacterium should not be too long. A bacterium can be considered as a single giant receptor insofar as the density of receptors on its surface is high. This is the case of the Adx autodisplay system, 180,000 active Adx molecules were found to be displayed $8.4 \mathrm{~nm}$ apart on the $17.2-\mu \mathrm{m}^{2}$ surface of a single cell [Jose and Meyer, 2007]. Around $10^{9}$ spherical bacteria of a diameter of $1 \mu \mathrm{m}$ could be contained in a volume of a couple of cubic millimetres. For a spherical bacterium of diameter y $\mu \mathrm{m}$, and diffusion distance $\mathrm{x}$, the time for the diffusion of the protein to a bacterium covered with receptors is linear in $\mathrm{y} / \mathrm{x}$ :

$$
<\mathrm{x}^{2}>=\mathrm{q}_{\mathrm{i}} \mathrm{Dt},
$$

where $\left\langle\mathrm{x}^{2}\right\rangle$ is the mean-square displacement (i.e. $\mathrm{x}$ is the mean distance from the starting point that a target will have diffused in time $t$ ); $q_{i}$ depends on dimensionality and is 6 for 3 dimensional diffusion; $\mathrm{D}$ is the diffusion coefficient (for a small protein in water $\mathrm{D}=10^{-6} \mathrm{~cm}^{2} \mathrm{~s}^{-1}$ ); $\mathrm{t}$ is time.

Suppose $\mathrm{x}=1 \mathrm{~mm}$ :

$10^{-2}=6 \times 10^{-6} \mathrm{t}$ so $\mathrm{t}=\left(10^{-2} \times 10^{6}\right) / 6 \mathrm{~s} \approx 10^{3} \mathrm{~s}$.

This does not take into account: (1) the blocking of the diffusing protein by the relatively static bacteria, or (2) the nonspecific binding of this protein to the wrong bacteria.

\section{A General Solution to Increase Sensitivity - Pruning the Bacterial RM Library}

A reduction in the size of the RM library (i.e. its variety) would help solve some of the problems of diffusion, nonspecific binding, and false-positives. This might be achieved by taking a leaf out of the human immune system in which those antibody-producing cells that react to self are eliminated early in the development of the system. In the case of the RM library, this would entail exposing the receptor library obtained in step 2 to antigens that would often be encountered. These antigens include those on the surface of the wild-type bacteria from which the library is derived as well as those found on common molecules that the user of the library is not interested in amplifying. How could this be achieved? One way would be to add the common antigens to the initial receptor population (fig. 3c) and discard all the bacteria that then fluoresced; a second way would be to select a phage library binding to these antigens and then to add these phage to the bacteria containing the peptide library with the hope that the phage might bind to the mimic peptide during its export and thereby infect and eliminate the bacterium.

\section{Example of an Application - The Prion}

Neurodegenerative disorders such as bovine spongiform encephalopathy are generally believed to be caused by the transmission of the aberrant conformation(s) of the disease-causing prion, $\mathrm{PrP}^{\mathrm{Sc}}$, to the normal cellular prion, $\mathrm{PrP}^{\mathrm{C}}$. Preventing and understanding these disorders depends on detecting the agents responsible and on obtaining their structures. Both detection and structural analysis pose problems. In raising antibodies to $\mathrm{Pr} \mathrm{P}^{\mathrm{Sc}}$, the authors write in a recent paper: "The development of antibodies with binding capacity towards soluble oligomeric forms of $\mathrm{PrP}^{\mathrm{Sc}}$, recognized in the aggregation process in early stage of the disease, would be of paramount importance in diagnosing prion diseases before extensive neuropathology has ensued. As blood transfusion appears to be efficient in the transmission of the infectious prion agent, there is an urgent need to develop reagents that would specifically recognize oligomeric forms of the abnormally folded prion protein, $\mathrm{PrP}^{\mathrm{Sc}}$ " [Tayebi et al., 2011]. The problem is also acute for structural analysis. Structural variations underpin the different properties of prion strains [Colby and Prusiner, 2011]. These variations include glycosylation, protease resistance, electrophoretic mobility and conformational stability. According to a recent review, the problem is that "the elucidation of atomic-level structures for these misfolded proteins has been nearly impossible, except in the case of short- to intermediate-length peptides. Major obstacles include insolubility, conformational heterogeneity, and difficulty in assembling misfolded proteins into crystals. Some structural insights have been achieved for fungal prions through solid-state NMR and hydrogen-exchange coupled with NMR. However, these observations depend on obtaining a high yield of the fungal prion proteins in their active state. Studies employing X-ray fiber diffraction, a technique that has yielded only low-resolution structural 
information... Whether the high-titer prions generated from recombinant PrP using sonication are of sufficient quality and quantity to allow for structural determination remains to be seen" [Colby and Prusiner, 2011].

How might MCR help? $\operatorname{PrP}^{\mathrm{C}}$ and various versions of $\mathrm{PrP}^{\mathrm{Sc}}$ might be used as target proteins in separate experiments to create specific RM libraries in which the RM bacteria recognize different epitopes and only fluoresce in the presence of the target (fig. 1). In the case of detection of $\mathrm{PrP}^{\mathrm{C}}$, the entire library (analogous to a polyclonal antibody) created for this target might then be used as the basis of a fluorescence assay to detect $\operatorname{PrP}^{\mathrm{C}}$ in a wide variety of samples. Moreover, the receptors in the RM bacteria might be purified and used, for example, in quantification of $\mathrm{PrP}^{\mathrm{C}}$ on Western blots. In the case of detection of $\mathrm{PrP}^{\mathrm{Sc}}$, the library created for this target would contain $\mathrm{RM}$ bacteria that would also recognize $\mathrm{PrP}^{\mathrm{C}}$. Such bacteria might be eliminated from the library by adding $\operatorname{PrP}^{\mathrm{C}}$, performing flow cytometry, and retaining only those bacteria that do not fluoresce (see 'A General Solution to Increase Sensitivity - Pruning the Bacterial RM Library'). The outcome of this molecular subtraction should be a $\mathrm{PrP}^{\mathrm{Sc}}$-specific library that could be used for both the detection and quantification of an aberrant prion. In the case of structural analyses [Legname, 2012], a precious contribution might be made by the existence of cognate $\mathrm{RM}$ pairs (some of which are specific to $\mathrm{PrP}^{\mathrm{C}}$, some to $\mathrm{PrP}^{\mathrm{Sc}}$, and some to both), since it should prove possible to determine the structure of both the mimic peptide and its complement in the receptor. Finally, MCR might be used to investigate factors in addition to prions that lead to neurodegeneration by, for example, creating an RM library to an extract from an infected specimen and then using an extract from an uninfected specimen to leave only RM bacteria specific to constituents of the infected extract; the reciprocal experiment might also be interesting.

\section{Discussion}

PCR has had a major impact on biology. A more general amplification technique, applicable to all types of target molecules, might be expected to have a similar impact. This would extend from the detection and identification of unknown target molecules to the generation in vitro of the equivalent of a set of antibodies to known molecules. In the latter case, it is possible that receptor/ mimic pairs (based on proteins and peptides) might have structures that would be easier to determine than those of antibody/antigens. This potential pay-off makes it worth exploring numerous speculative approaches and the problems they raise. These problems include those of noise, diffusion, nonspecific binding, and the propensity of bacteria to mutate, recombine and rearrange their genomes. The strategy we favor is one in which a bacterial population displays on the surface of each bacterium a receptor that binds to a different epitope; on binding to the epitope the receptor then acts as a sensor kinase that signals to a response regulator to turn on production (and possibly release from an intracellular store) of a peptide that mimics the epitope. This constitutes an autoinduction. We choose this strategy because displaying a protein library on the surface of bacteria is known to confer advantages that include accessibility, stability, and relatively easy purification of the receptor and selection of the corresponding gene [Jose et al., 2005]. We couple surface display with the system of sensor kinase and response regulator because such systems are highly specific and modular [Ninfa, 2010].

Depending on the use to which MCR would be put, some of the problems that confront the technique are less serious than they first appear and, as in the case of generating antibodies, diffusion for example is not a problem if the target can be purified in a sufficient quantity. Some of these problems may also have confronted the human immune system itself. It may therefore be worth considering adopting a similar solution, namely the elimination of certain antibodies early in development. This would lead to MCR being a kind of in vitro bacterial immune system.

There are, potentially, a great many ways to implement the scheme for the library construction outlined above. Some may work and many will not. The need for this extensive exploration is one reason why the general scientific community needs to be involved. At every stage in the development of MCR there are several alternative possibilities. Neither receptor nor mimic are limited to proteins and peptides and DNA aptamers, for example, offer an attractive alternative. Indeed, if the mimic were a 'smart aptamer' based on DNA rather than protein, a wide range of RM pairs could be selected with dissociation constants of, for example, $10^{-8}$ [Drabovich et al., 2009]. In the case of the prion as target, it might be interesting to generate a peptide library using part or all of the sequence of the prion itself thereby exploiting its capacity to self-assemble into fibers. The amplifier could be one of several types of cells, including eukaryotic cells and the minimal cells or cell-like systems that are pursued by synthetic biologists. Detection does not have to be per- 
formed with fluorescence nor does selection have to use an antibiotic or FACS. The sensor-regulator system could take a multitude of forms. Construction of the receiver and peptide libraries could be separate, as presented here, but they could be constructed together. The possibilities are sufficient to engage a variety of scientists from different fields for many years. The effort will be worth it if as we believe, MCR is indeed on the horizon.

\section{Acknowledgements}

We thank the anonymous referees for helpful comments and, for support, we thank the Epigenomics project, Genopole, the CNRS (DYCOEC, GDR 2984 and the European Network in Systems Biology, GDRE 513).

\section{References}

Carmany DO, Hollingsworth K, McCleary WR: Genetic and biochemical studies of phosphatase activity of phor. J Bacteriol 2003;185: 1112-1115.

Colby DW, Prusiner SB: De novo generation of prion strains. Nat Rev Microbiol 2011;9:771777.

-Drabovich AP, Berezovski MV, Musheev MU, Krylov SN: Selection of smart small-molecule ligands. The proof of principle. Analyt Chem 2009;81:490-494.

- Hammar P, Leroy P, Mahmutovic A, Marklund EG, Berg OG, Elf J: The lac repressor displays facilitated diffusion in living cells. Science 2012;336:1595-1598.

Hastings JW, Greenberg EP: Quorum sensing: the explanation of a curious phenomenon reveals a common characteristic of bacteria. J Bacteriol 1999;181:2667-2668.

-Hense BA, Kuttler C, Muller J, Rothballer M, Hartmann A, Kreft JU: Does efficiency sensing unify diffusion and quorum sensing? Nat Rev Microbiol 2007;5:230-239.

-Jose J, Betscheider D, Zangen D: Bacterial surface display library screening by target enzyme labeling: Identification of new human cathepsin g inhibitors. Anal Biochem 2005; 346:258-267.

Jose J, Meyer TF: The autodisplay story, from discovery to biotechnical and biomedical applications. Microbiol Mol Biol Rev 2007;71: 600-619.
Legname G: Early structural features in mammalian prion conformation conversion. Prion 2012;6:37-39.

-Mouliere F, Robert B, Arnau Peyrotte E, Del Rio M, Ychou M, Molina F, Gongora C, Thierry AR: High fragmentation characterizes tumour-derived circulating DNA. PloS One 2011;6:e23418.

Ninfa AJ: Use of two-component signal transduction systems in the construction of synthetic genetic networks. Curr Opin Microbiol 2010;13:240-245.

Novick RP, Geisinger E: Quorum sensing in staphylococci. Annu Rev Genet 2008;42: 541-564.

Pazgier M, Liu M, Zou G, Yuan W, Li C, Li C, Li J, Monbo J, Zella D, Tarasov SG, Lu W: Structural basis for high-affinity peptide inhibition of $\mathrm{p} 53$ interactions with $\mathrm{mdm} 2$ and mdmx. Proc Natl Acad Sci USA 2009;106: 4665-4670

Perego M: Kinase-phosphatase competition regulates Bacillus subtilis development. Trends Microbiol 1998;6:366-370.

Perelson AS, Oster GF: Theoretical studies of clonal selection: minimal antibody repertoire size and reliability of self-non-self discrimination. J Theoret Biol 1979;81:645670 .
- Posfai G, Plunkett III G, Feher T, Frisch D, Keil GM, Umenhoffer K, Kolisnychenko V, Stahl B, Sharma SS, de Arruda M, Burland V, Harcum SW, Blattner FR: Emergent properties of reduced-genome Escherichia coli. Science 2006;312:1044-1046.

-Skerker JM, Perchuk BS, Siryaporn A, Lubin EA, Ashenberg O, Goulian M, Laub MT: Rewiring the specificity of two-component signal transduction systems. Cell 2008;133:10431054.

Tayebi M, Jones DR, Taylor WA, Stileman BF, Chapman C, Zhao D, David M: Prp(sc)-specific antibodies with the ability to immunodetect prion oligomers. PloS One 2011; 6:e19998

Turovskiy Y, Kashtanov D, Paskhover B, Chikindas ML: Quorum sensing: fact, fiction, and everything in between. Adv Appl Microbiol 2007;62:191-234.

-Utsumi R, Brissette RE, Rampersaud A, Forst SA, Oosawa K, Inouye M: Activation of bacterial porin gene expression by a chimeric signal transducer in response to aspartate. Science 1989;245:1246-1249.

Yamaguchi Y, Park JH, Inouye M: Mqsr, a crucial regulator for quorum sensing and biofilm formation, is a GCU-specific mRNA interferase in Escherichia coli. J Biol Chem 2009; 284:28746-28753. 